\title{
COUPLED OPTICS RECONSTRUCTION FROM TBT DATA USING MAD-X*
}

\author{
Y. Alexahin, E. Gianfelice-Wendt (FNAL, Batavia, IL), \\ V. Kapin (MEPhI, Moscow, Russia), F. Schmidt (CERN, Geneva, Switzerland)
}

\section{Abstract}

Turn-by-turn BPM data provide immediate information on the coupled optics functions at BPM locations. In the case of small deviations from the known (design) uncoupled optics some cognizance of the sources of perturbation, BPM calibration errors and tilts can also be inferred without detailed lattice modeling. In practical situations, however, fitting the lattice model with the help of some optics code would lead to more reliable results. We present an algorithm for coupled optics reconstruction from TBT data on the basis of MAD-X and give examples of its application for the Fermilab Tevatron accelerator.

\section{INTRODUCTION}

Precise knowledge of the beam optics is a prerequisite for successful performance of an accelerator. It is important to have tools for measurement and correction of beta-beating, coupling, for detection of sources of optics imperfections. There is a number of methods for optics measurement - ORM, AC dipole, TBT - of which the turn-by-turn (TBT) method looks preferable since it provides immediate information on the eigenmodes of betatron oscillations.

Some information on sources of optics perturbation, as well as BPM calibration errors and tilts can be inferred from the TBT data using perturbation theory without detailed lattice modeling (see e.g. Ref.[1]). However, this approach fails in the presence of both focusing and BPM errors which are typical for interaction regions in colliders.

To disentangle the effect of optics perturbation from BPM errors and pinpoint the sources of perturbations it is necessary to construct a lattice model and fit its parameters to the measurement data. The code for matching must handle the coupled optics case since the working point of most accelerators is close to the diagonal to allow space for various tune shifts.

MAD-X [2] (unlike its predecessor MAD-8) is capable of matching coupled optics and - which is no less important - allows user-defined expressions in matching constraints. In this report we show how to use MAD-X for coupled optics reconstruction and determination of BPM calibration errors and tilts from TBT data.

\section{BASIC RELATIONS}

\section{Coupled motion description in MAD}

There are two representations of coupled optics functions available in MAD-X: the Mais-Ripken

\footnotetext{
* Work supported by Fermi Research Alliance, LLC under Contract
} DE-AC02-07CH11359 with the U.S. DOE. functions [3] computed by PTC_TWISS command and the Edwards-Teng functions [4] computed by the kernel TWISS command. Based on these representations two versions of the program were developed [5]. Though the Mais-Ripken parameterization is more suitable for TBT data analysis it requires loading the PTC module increasing occupied memory and computation time which may be critical for a console application. Therefore we present here only the version employing the EdwardsTeng parameterization.

Coupled transverse oscillations of a particle can be described as

$$
\left(\begin{array}{l}
x \\
x^{\prime} \\
y \\
y^{\prime}
\end{array}\right)=\sum_{m=1,2} \underline{\mathrm{V}}_{m} \cdot\left(\begin{array}{l}
a_{m}^{\prime} \cos \varphi_{m}-a_{m}^{\prime \prime} \sin \varphi_{m} \\
a_{m}^{\prime} \sin \varphi_{m}+a_{m}^{\prime \prime} \cos \varphi_{m}
\end{array}\right)
$$

where $a_{m}=a_{m}^{\prime}+i a_{m}^{\prime \prime}$ are constants of the motion changing at the origin by a factor $\exp \left(2 \pi i Q_{m}\right)$ from one turn to another, $\varphi_{m}=2 \pi \mu_{m}$ are betatron phase advances $\left(Q_{m} \equiv \mu_{m}(C)\right), \underline{\underline{V}}_{m}$ are real $4 \times 2$ matrices which can be expressed via the Edwards-Teng optics functions and $2 \times 2$ coupling matrix $\boldsymbol{R}$ also computed by the TWISS command $^{\dagger}$ as

$$
\begin{aligned}
& \underline{\mathrm{V}}_{1}=(1+\operatorname{det} \boldsymbol{R})^{-1 / 2}\left(\begin{array}{c}
\boldsymbol{V}_{1} \\
-\boldsymbol{R} \boldsymbol{V}_{1}
\end{array}\right), \\
& \underline{\mathrm{V}}_{2}=(1+\operatorname{det} \boldsymbol{R})^{-1 / 2}\left(\begin{array}{c}
\overline{\boldsymbol{R}} \boldsymbol{V}_{2} \\
\boldsymbol{V}_{2}
\end{array}\right),
\end{aligned}
$$

where $\overline{\boldsymbol{R}}=-\boldsymbol{S} \boldsymbol{R} \boldsymbol{S}$ is the symplectic conjugate to $\boldsymbol{R}$ and

$$
\boldsymbol{V}_{m}=\left(\begin{array}{cc}
\beta_{m}^{1 / 2} & 0 \\
-\alpha_{m} \beta_{m}^{-1 / 2} & -\beta_{m}^{-1 / 2}
\end{array}\right)
$$

with $\alpha_{m}, \beta_{m}, m=1,2$ being Twiss parameters for the two normal modes of oscillations.

From BPMs we have information only on the beam position so only the spatial components of eq.(1) will be used which we rewrite in the form ${ }^{\ddagger}$ :

$$
\left(\begin{array}{l}
x \\
y
\end{array}\right)=\left(\begin{array}{l}
V_{11} \\
V_{31}
\end{array}\right) a_{1}^{\prime}-\left(\begin{array}{l}
V_{12} \\
V_{32}
\end{array}\right) a_{1}^{\prime \prime}+\left(\begin{array}{l}
V_{13} \\
V_{33}
\end{array}\right) a_{2}^{\prime}-\left(\begin{array}{c}
V_{14} \\
V_{34}
\end{array}\right) a_{2}^{\prime \prime}
$$

where $V_{k l}$ are real and imaginary components of the transfer matrix eigenvectors which can be expressed via the Mais-Ripken functions as

\footnotetext{
${ }^{\dagger}$ MAD Physicist's Guide gives different definition for $\boldsymbol{R}$ from actually used in MAD.

${ }^{\ddagger}$ Please note that $V_{k l}$ are not the elements of matrices from eq.(1).
} 


$$
\begin{aligned}
& V_{1,2 m-1}=\sqrt{\beta_{x m}} \cos \Phi_{x m}, V_{1,2 m}=\sqrt{\beta_{x m}} \sin \Phi_{x m}, \\
& V_{3,2 m-1}=\sqrt{\beta_{y m}} \cos \Phi_{y m}, V_{3,2 m}=\sqrt{\beta_{y m}} \sin \Phi_{y m} .
\end{aligned}
$$

From eqs. (1)-(5) follow relations between the MaisRipken and Edwards-Teng functions

$$
\begin{aligned}
\beta_{x \mathrm{I}} & =\kappa \beta_{x}, \beta_{y \mathrm{II}}=\kappa \beta_{y}, \kappa=(1+\operatorname{det} \boldsymbol{R})^{-1}, \\
\beta_{x \mathrm{II}} & =\kappa\left[R_{22}\left(R_{22} \beta_{y}+2 R_{12} \alpha_{y}\right)+R_{12}^{2} \gamma_{y}\right], \\
\beta_{y \mathrm{I}} & =\kappa\left[R_{11}\left(R_{11} \beta_{x}-2 R_{12} \alpha_{x}\right)+R_{12}^{2} \gamma_{x}\right], \\
\Phi_{x \mathrm{I}} & =\varphi_{x}, \Phi_{y \mathrm{II}}=\varphi_{y}, \\
\Phi_{x \mathrm{II}} & =\varphi_{y}-\arctan \left[R_{12} /\left(R_{22} \beta_{y}+R_{12} \alpha_{y}\right)\right], \\
\Phi_{y \mathrm{I}} & =\varphi_{x}+\arctan \left[R_{12} /\left(R_{11} \beta_{x}-R_{12} \alpha_{x}\right)\right] .
\end{aligned}
$$

\section{BPM errors}

Besides electronics errors there are orbit drifts leading to variation in differential BPM response so the BPM calibration factors should be corrected for every measurement. Defining the calibration factors as the ratio $r=x_{\text {actual }} / x_{\text {reported }}$ and taking also into account possible BPM tilts $\chi$ we can write for BPM readings

$$
x^{(r e p)}=\frac{x+y \tan \chi}{r}, y^{(r e p)}=\frac{y-x \tan \chi}{r}
$$

\section{ALGORITHM FOR OPTICS RECONSTRUCTION}

We assume here that data for both normal modes is available which is usually the case.

\section{TBT data processing}

The first step is to analyze the TBT data by the master program into the normal modes which can be done along the lines presented in Ref.[1]. Components $V_{k l}$ at horizontal and vertical BPMs can be obtained by separating real and imaginary parts in equations

$$
\begin{aligned}
& V_{1,2 m-1}^{(T B T)}+i V_{1,2 m}^{(T B T)}=\frac{2}{N a_{m}} \sum_{n=1}^{N} e^{-2 \pi i n Q_{m}} x_{n}^{(r e p)}, \\
& V_{3,2 m-1}^{(T B T)}+i V_{3,2 m}^{(T B T)}=\frac{2}{N a_{m}} \sum_{n=1}^{N} e^{-2 \pi i n Q_{m}} y_{n}^{(r e p)},
\end{aligned}
$$

Complex amplitudes are not known in advance exactly. Their absolute values are equivalent to common factors in the BPM calibration constants which can be adjusted during the fitting process by requiring the average value of the calibration constants for BPMs of each orientation to be one. The argument of $a_{m}$ can be found from the requirement that the corresponding betatron phase advance be zero at the lattice starting point which is automatically assumed in MAD. To preserve the balance between the number of variables and constraints we impose the requirement on the tunes to match the measured ones.

Initial values of complex amplitudes $a_{m}$, BPM calibration factors and tilts can be found with the help of perturbation theory as described in Ref.[1].

\section{MAD-X macros for eigenvectors}

First, the expressions for eigenvectors components $\mathrm{V}_{k l}$ should be introduced. We do this in two steps: express them via the Mais-Ripken functions according to eqs.(5) which are then replaced by the Edwards-Teng functions using eqs. (6):

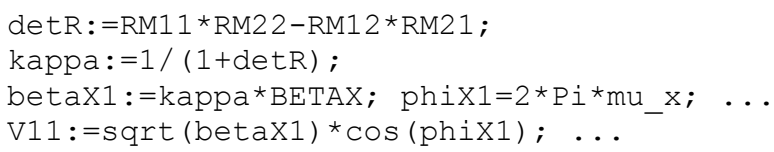

Also, MAD should be told where to take the values of primary functions from:

BETAX:=table (twiss, BETX);
mu_x:=table (twiss, MUX);
$\operatorname{RM1} 1:=$ table (twiss, R11); ...

Now we can build a macro-command which will fill the columns of the TWISS table with the desired functions:

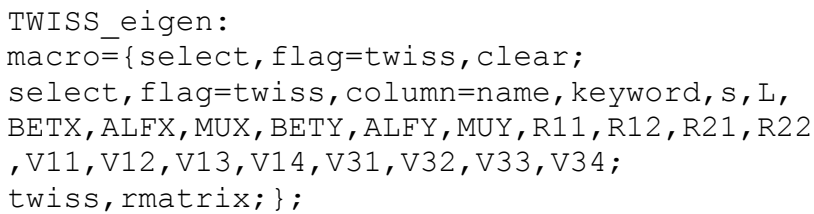

\section{Matching procedure}

Next, we should name the values of eigenvectors at BPMs to use them in constraints and show from which row of the TWISS table they should be taken, e.g. for the BPM named M01:

$$
\text { V11_M01:=table (twiss, M01,V11); ... }
$$

The matching module runs as

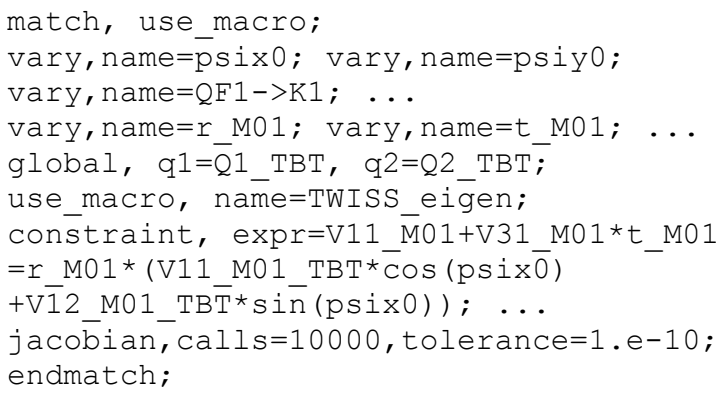

where psixo and psiyo are corrections to the phase of $a_{1}$ and $a_{2}$ respectively.

\section{TEVATRON INJECTION LATTICE TEST}

The above described fitting procedure has been tested using as target values the eigenvector components generated with MAD-X for the Tevatron injection optics. Then the strengths of two skew quadrupoles were changed from their nominal values and a tilt of $0.01 \mathrm{rad}$ was introduced for one of the horizontal BPMs prior to fitting. Using 11 skew quadrupoles and the tilt of all 118 
horizontal BPMs as variables, MAD-X was able to find in 217 iterations with high accuracy the correct values for all 11 skew quadrupoles (see Fig.1) and the original tilt of all BPMs.

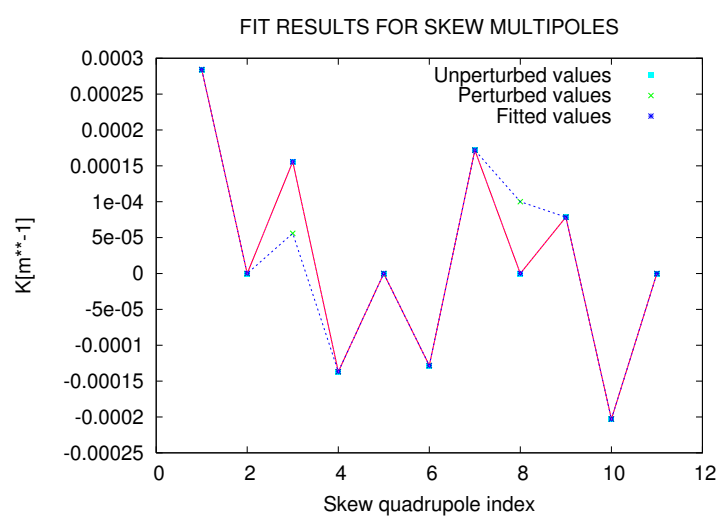

Figure 1: Algorithm test

\section{SUMMARY AND OUTLOOK}

Method of optics reconstruction proposed in this report is potentially a powerful tool. However, the fitting procedure for a machine as complicated as the TEVATRON takes too long time to be used in online applications. One step of full optics reconstruction which requires fitting over 900 parameters takes more than one hour on a $2 \mathrm{GHz} \mathrm{PC}$.

There are several possibilities to speed up the calculations. The first one involves the internal MAD-X matching procedure. The TEVATRON lattice version used in our preliminary tests consists of 13260 elements. The TWISS command of the current MAD-X code tracks the lattice optical functions via the element-by-element advancing, and therefore it is very time consuming for such large rings. The discussed optics reconstruction algorithm deals with a small subset of lattice elements, namely, for TEVATRON, 236 BPMs and 432 variable quadrupoles simulating focusing errors, while other elements between them are unchanged and can be replaced by equivalent sector maps. This may reduce the computation time by an order of magnitude.

Some gain in computation speed can be achieved by simply removing unnecessary elements from the machine lattice file.

One more possibility for significant reduction in the computation time with the present version of MAD-X is to divide the matching process in two parts: 1) MAD fitting the magnetic element strengths as described here with fixed values of BPM calibration factors and tilts; 2) adjustment of the calibration factors and tilts by the master program on the basis of simple least square fit and providing these values to MAD for the next iteration. This will eliminate the unnecessary and time consuming numerical computation of derivatives w.r.t. the BPM parameters.

\section{ACKNOWLEDGEMENTS}

The authors would like to thank N.Gelfand for help with conversion of the TEVATRON lattice files into the MAD-X format.

\section{REFERENCES}

[1] Y. Alexahin, E.Gianfelice-Wendt, FERMILAB-PUB06-093-AD, 2006.

[2] "MAD-X User Guide"' http://mad.home.cern.ch/ mad/uguide.html

[3] G. Ripken, F. Willeke, "Methods of Beam Optics", DESY 88-114, 1988.

[4] D.A. Edwards, L.C. Teng, IEEE Trans. NS, 20 (1973), pp. 885-888.

[5] Y.Alexahin,V. Kapin, F. Schmidt, FNAL Beamsdoc-2449-v2 (2006). http://beamdocs.fnal.gov/ADpublic/DocDB/DocumentDatabase 